\title{
CaMKII $\delta$ overexpression in hypertrophy and heart failure: cellular consequences for excitation-contraction coupling
}

\section{L.S. Maier}

Correspondence

L.S. Maier

Abteilung Kardiologie and

Pneumologie,

Herzzentrum

Georg-August-Universität Göttingen

Robert-Koch-Str. 40

37075 Göttingen

Germany

Fax: +49-551-39-8941

E-mail: Imaier@med.uni-goettingen.de Internet: www.Imaier.de

Part of a talk presented at the XIX Annual Meeting of the

Federação de Sociedades de Biologia Experimental, Águas de Lindóia, SP, Brazil, August 25-29, 2004.

L.S. Maier is the recipient of a grant from the Deutsche

Forschungsgemeinschaft (DFG, Emmy Noether-program, MA 1982/2-1 and MA 1982/4-1), a Young Investigator Award from the GlaxoSmithKline Research Foundation,

and a grant from the University of Göttingen (Anschubfinanzierung).

Received January 31, 2005

Accepted May 5, 2005

.................
Abteilung Kardiologie and Pneumologie, Herzzentrum, Georg-August-Universität Göttingen, Göttingen, Germany

\begin{abstract}
$\mathrm{Ca} /$ calmodulin-dependent protein kinase II $\delta$ (CaMKII $\delta$ ) is the predominant isoform in the heart. During excitation-contraction coupling (ECC) CaMKII phosphorylates several Ca-handling proteins including ryanodine receptors (RyR), phospholamban, and L-type Ca channels. CaMKII expression and activity have been shown to correlate positively with impaired ejection fraction in the myocardium of patients with heart failure and CaMKII has been proposed to be a possible compensatory mechanism to keep hearts from complete failure. However, in addition to these acute effects on ECC, CaMKII was shown to be involved in hypertrophic signaling, termed excitation-transcription coupling (ETC). Thus, animal models have shown that overexpression of nuclear isoform $\mathrm{CaMKII}_{\mathrm{B}}$ can induce myocyte hypertrophy. Recent study from our laboratory has suggested that transgenic overexpression of the cytosolic isoform $\mathrm{CaMKII}_{\mathrm{C}}$ in mice causes severe heart failure with altered intracellular $\mathrm{Ca}$ handling and protein expression leading to reduced sarcoplasmic reticulum (SR) Ca content. Interestingly, the frequency of diastolic spontaneous SR Ca release events (or opening of RyR) was greatly enhanced, demonstrating increased diastolic SR Ca leak. This was attributed to increased CaMKII-dependent RyR phosphorylation, resulting in increased and prolonged openings of RyR since Ca spark frequency could be reduced back to normal levels by CaMKII inhibition. This review focuses on acute and chronic effects of CaMKII in ECC and ETC. In summary, CaMKII overexpression can lead to heart failure and CaMKII-dependent RyR hyperphosphorylation seems to be a novel and important mechanism in ECC due to SR Ca leak which may be important in the pathogenesis of heart failure.
\end{abstract}

Key words - Calcium

- Calmodulin

- CaM kinase

- Excitation-contraction

coupling

- Heart

\section{Structure of $\mathrm{Ca} /$ calmodulin protein kinase II}

$\mathrm{Ca} /$ calmodulin protein kinase II (CaMKII) is a multifunctional serine/threonine protein kinase mainly found in the heart $(1,2)$ which can phosphorylate several proteins in response to increasing intracellular $\mathrm{Ca}$ concentrations, $[\mathrm{Ca}]_{\mathrm{i}}$ (3). There are four different CaMKII genes $(\alpha, \beta, \gamma, \delta)$ of which CaMKII $\delta$ protein is the most abundant isoform in the heart $(1,4)$. Subcellular localizations of CaMKII $\delta$ were 
found, with $\delta_{\mathrm{B}}$ being specifically compartmentalized to the nucleus by means of an 11amino acid long nuclear localization sequence and with $\delta_{\mathrm{C}}$ being the cytosolic isoform without nuclear localization sequence (4). Each CaMKII isoform contains a catalytic domain, a central regulatory domain (containing partially overlapping autoinhibitory and CaM binding regions) and an association domain responsible for oligomerization $(1,2)$. When $\mathrm{Ca} / \mathrm{CaM}$ binds to the regulatory region (amino acid 296-311) the active site on the catalytic domain gains access to target substrates.

The CaMKII holoenzyme consists of homo- or heteromultimers of 6-12 kinase subunits forming a wheel-like structure $(1,3)$. During CaMKII activation, $\mathrm{Ca} / \mathrm{CaM}$ binds to the regulatory domain and displaces the autoinhibitory domain (amino acid 273-309) on CaMKII thereby activating the enzyme with a half maximal activation at $[\mathrm{Ca}]_{\mathrm{i}}$ of 500-1000 nM (i.e., systolic $[\mathrm{Ca}]_{\mathrm{i}}$ ). Subsequently, the kinase locks itself into an activated state by autophosphorylation of Thr287 on the autoinhibitory segment (4). Autophosphorylation is critical in maintaining the enzyme active even after $[\mathrm{Ca}]_{i}$ has declined and $\mathrm{Ca} / \mathrm{CaM}$ has dissociated from its binding region (autonomous state). It is important to know that autophosphorylation itself is not essential for initial CaMKII activity, but does have important consequences, i.e., by increasing the affinity of the kinase$\mathrm{CaM}$ complex (5). This effect traps $\mathrm{Ca} / \mathrm{CaM}$ in the autophosphorylated subunit. At high $[\mathrm{Ca}]_{i}$, the affinity of $\mathrm{Ca} / \mathrm{CaM}$ for CaMKII increases $~ 700$-fold from $45 \mathrm{nM}$ to $60 \mathrm{pM}$ $(1,5)$. Even when $[\mathrm{Ca}]_{\mathrm{i}}$ declines to resting levels during diastole (i.e., 100 nM), CaM is still trapped for several seconds. As a result, the kinase retains $100 \%$ activity as long as CaM is trapped, regardless of the $[\mathrm{Ca}]_{\mathrm{i}}$ level (5). Interestingly, autophosphorylation is sufficient to disrupt the autoinhibitory domain, with the enzymes remaining partially active even after $\mathrm{CaM}$ has dissociated from this autonomous state (20-
$80 \%$ ) (6-9). For complete inactivation to occur, CaMKII can be dephosphorylated by protein phosphatases including PP1, PP2A, and PP2C (4).

Several CaMKII inhibitors have been widely used in cardiac myocytes, including the organic inhibitors KN62 and KN93 (2) which competitively inhibit CaM binding at the regulatory domain to CaMKII $\left(\mathrm{K}_{\mathrm{i}} \sim 0.37\right.$ $\mu \mathrm{M})$ and are quite selective. Unfortunately, some of these agents appear to have direct ion channel effects which may be independent of CaMKII action $(10,11)$. In contrast, peptide inhibitors are not known to affect ion channels. Two useful peptides are autocamtide-2related inhibitory peptide (AIP) with 13 amino acids (12), and autocamtide-2 inhibitory peptide (AC3-I; Ref. 13).

\section{CaMKII and excitation-contraction coupling}

During excitation-contraction coupling (ECC) $\mathrm{Ca}$ enters the cell mainly via voltagedependent L-type Ca channels $\left(\mathrm{I}_{\mathrm{Ca}}\right)$, triggering $\mathrm{Ca}$ release from the sarcoplasmic reticulum (SR) via SR Ca channels (ryanodinereceptors, RyR), a process termed Ca-induced $\mathrm{Ca}$ release (14). These processes increase intracellular $[\mathrm{Ca}]_{\mathrm{i}}$, causing $\mathrm{Ca}$ binding to troponin $\mathrm{C}$ which activates the myofilaments leading to contraction (systole). For diastolic relaxation to occur, Ca must be removed from the cytoplasm. The SR CaATPase (SERCA) and the $\mathrm{Na} / \mathrm{Ca}$-exchanger (NCX) are the main mechanisms for $\mathrm{Ca}$ removal (14). CaMKII can modulate ECC by phosphorylating several important $\mathrm{Ca}$ regulatory proteins in the heart in response to $\mathrm{Ca}$ signals, including RyR $(15,16)$ and phospholamban (PLB; Refs. 17,18), and possibly L-type Ca channels (2), with multiple functional consequences.

\section{Facilitation of $\mathrm{I}_{\mathrm{Ca}}$}

CaMKII modulates L-type Ca channels 
and thereby $\mathrm{I}_{\mathrm{Ca}}$, and this is most clearly seen functionally as a positive staircase of $\mathrm{I}_{\mathrm{Ca}}$ with repeated depolarization from -90 to 0 $\mathrm{mV}$, a process termed facilitation $(19,20)$. $\mathrm{I}_{\mathrm{Ca}}$ amplitude increases and inactivation is slowed progressively over $2-5$ pulses at 1 Hz. Several groups independently demonstrated that Ca-dependent $\mathrm{I}_{\mathrm{Ca}}$ facilitation is mediated by CaMKII-dependent phosphorylation (21-23). At the single channel level this CaMKII-dependent $\mathrm{I}_{\mathrm{Ca}}$ facilitation is manifest as longer single channel openings (24). This positive $\mathrm{I}_{\mathrm{Ca}}$ staircase is Ca-dependent, very local at the mouth of the Ltype $\mathrm{Ca}$ channels, and still occurs in the absence of SR Ca release, since it is still observed when cells are heavily Ca buffered with $10 \mathrm{mM}$ EGTA. The physiological role of $\mathrm{I}_{\mathrm{Ca}}$ facilitation is not entirely clear, but it may partly offset reduced L-type Ca channel availability at high heart rates (caused by direct $\mathrm{Ca}$-dependent inactivation).

\section{Sarcoplasmic reticulum Ca release}

CaMKII also affects RyR activity. Based on sequence analysis, there are up to four serine and two threonine residues at the RyR that are consensus phosphorylation sites. Witcher et al. (15) first reported direct phosphorylation of cardiac RyR at Ser-2809 by CaMKII activating the SR Ca release channel. Additional studies also showed that RyR are substrates of CaMKII $(16,25)$, but the specific effects of phosphorylation reported in these studies remain controversial. That is, CaMKII either increases $(15,16)$ or decreases RyR open probability (25). Most of the work on the effects of CaMKII on RyR was conducted using RyR in lipid bilayers or by measuring Ca release from SR vesicles, but few data have been obtained for more physiological environments. Li et al. (10) reported that in intact voltage-clamped cardiac myocytes endogenous CaMKII increased the amount of SR Ca release for a given SR Ca content and $\mathrm{I}_{\mathrm{Ca}}$ trigger. In that study, the effect of CaMKII on RyR was evaluated when both L-type Ca current and SR Ca load were constant under control conditions and in the presence of the CaMKII inhibitor $\mathrm{KN}-93$. This conclusion is also consistent with observations that protein phosphates (PP1 and PP2A) can reduce RyR activity for a given $\mathrm{I}_{\mathrm{Ca}}$ and SR Ca load, and that, conversely, phosphatase inhibitors enhance it (26). However, Wu et al. (27) obtained opposite results suggesting that constitutively active CaMKII inhibited SR Ca release, while CaMKII inhibition (by AC3I) enhanced SR Ca release. Since they did not measure SR Ca content in the same protocols, the dichotomy raised is still unresolved.

Exciting reports from our laboratory and others over the past two years have provided new evidence in isolated cardiac myocytes that CaMKII indeed is directly associated with RyR (28) and overexpression of CaMKII increases SR Ca release as shown by increased spontaneous SR Ca release events termed $\mathrm{Ca}$ spark frequency (see in detail below: CaMKII and hypertrophy and heart failure). In contrast, when blocking CaMKII (using KN-93) Ca spark frequency decreases dramatically (29). These results in myocytes from CaMKII transgenic mouse hearts were confirmed recently by Currie et al. (30) showing that the CaMKII peptide inhibitor AIP (1 $\mu \mathrm{M})$ depresses Ca spark frequency in rabbit hearts due to decreased endogenous CaMKII binding to RyR. Interestingly, Wehrens et al. (31), using site-directed mutagenesis, most recently showed that CaMKII binding at RyR might not be at Ser-2809 (protein kinase A, PKA, site with subsequent FKBP12.6 dissociation) but at Ser-2815. In addition, they showed that CaMKII-dependent RyR activation resulted in even more active RyR compared to PKA-dependent RyR phosphorylation. They clearly showed that CaMKIIdependent RyR phosphorylation increased RyR open probability using single channel measurements in lipid bilayers (31). Inter- 
estingly, the exact mechanism of RyR activation remained unclear since FKBP12.6 dissociation (as seen by PKA-dependent phosphorylation) does not seem to occur.

\section{Frequency-dependent acceleration of relaxation}

PLB is an endogenous inhibitor of SERCA in its unphosphorylated state (32). Upon PLB phosphorylation SERCA activity and SR Ca uptake are enhanced. PLB can be phosphorylated by PKA at Ser-16 and by CaMKII at Thr-17 $(18,32)$ with a reduction of $K_{\mathrm{m}}(\mathrm{Ca})$ of SERCA similar to Ser-16 phosphorylation by PKA. Although less generally accepted, there are also reports that suggest an increase of $\mathrm{V}_{\text {max }}$ due to CaMKII phosphorylation of PLB with less effect on $K_{\mathrm{m}} v s$ PKA. Bassani et al. (33) showed that CaMKII phosphorylation of PLB might be responsible for the frequency-dependent acceleration of relaxation (FDAR) of twitches and SR Ca uptake typically seen in intact myocytes. Hagemann et al. (34) recently showed a frequency-dependent increase in PLB Thr-17 phosphorylation in rat myocytes even in the absence of Ser-16 phosphorylation, and that the level of CaMKIIdependent Thr-17 phosphorylation correlated with the rate of relaxation.

Physiologically, FDAR may be an important intrinsic mechanism to allow faster relaxation (and diastolic filling) when heart rate is increased. FDAR is also manifest as slowing of twitch relaxation as time between beats is prolonged (i.e., at post-rest contractions; Ref. 33). As mentioned above, FDAR is also reflected on the rate of $[\mathrm{Ca}]_{i}$ decline and is attributable to altered SR Ca uptake (33). Schouten (35) proposed that FDAR might be due to enhanced SR Ca uptake via PLB phosphorylation by CaMKII, activated by the cyclic increase in $[\mathrm{Ca}]_{\mathrm{i}}$ during ECC. Conversely, rest would allow deactivation of CaMKII and dephosphorylation of PLB, reversing the stimulation of SR-dependent
$[\mathrm{Ca}]_{\mathrm{i}}$ decline. In intact rat myocytes, the CaMKII inhibitor KN-62 prevents the acceleration of $[\mathrm{Ca}]_{\mathrm{i}}$ decline in steady-state vs post-rest twitches, whereas phosphatase inhibitors prevent the slowing of $[\mathrm{Ca}]_{\mathrm{i}}$ decline at the post-rest twitch (33). While PLB is a logical CaMKII target explaining FDAR, we found that FDAR is still quite prominent in PLB-deficient mice and still sensitive to CaMKII inhibition by KN-93 (36). Thus, while PLB might contribute to FDAR, it cannot be the sole mechanism. There have been several reports of direct CaMKII-dependent phosphorylation of cardiac SERCA, and increased $\mathrm{V}_{\max }$ for $\mathrm{Ca}$ transport; Refs. 2,37). While this might fit with FDAR results above, critical studies have shown that CaMKII does not directly phosphorylate SERCA2 $(2,37)$. Thus, the identity of the CaMKII target involved in accelerating SR $\mathrm{Ca}$ transport during FDAR has not yet been clearly determined.

\section{Acidosis}

Acidosis, a substantial decrease in intracellular $\mathrm{pH}\left(\mathrm{pH}_{\mathrm{i}}\right)$, depresses myocardial contractility mainly due to decreased myofilament Ca sensitivity (37). During long periods of acidosis, there is a progressive increase in $\Delta[\mathrm{Ca}]_{\mathrm{i}}$ causing a partial recovery of contractions (37). Increased $[\mathrm{H}]_{\mathrm{i}}$ during acidosis displaces $\mathrm{Ca}$ ions from proteins (e.g., troponin $\mathrm{C}$ ), thereby raising resting (diastolic) $[\mathrm{Ca}]_{\mathrm{i}}$ (37). The combination of increased $\Delta[\mathrm{Ca}]_{\mathrm{i}}$ and diastolic $[\mathrm{Ca}]_{\mathrm{i}}$ may contribute to the recovery of contractility. Also, an increased SR Ca content contributes to this recovery which is probably due to a combination of higher diastolic $[\mathrm{Ca}]_{\mathrm{i}}$, inhibition of SR Ca release, elevated $[\mathrm{Na}]_{i}$, and $[\mathrm{Ca}]_{\mathrm{i}}(37)$. The increase in SR Ca load may gradually increase $[\mathrm{Ca}]_{i}$ transients and offsets a reduced SR Ca fractional release at low $\mathrm{pH}$ (37).

Inhibition of SR Ca-ATPase during acidosis is manifest by the slowed decline of $\mathrm{Ca}$ 
transients and contractions, which progressively recover during late acidosis and are accelerated concomitant with an increase in the amplitudes of $[\mathrm{Ca}]_{\mathrm{i}}$ transients and contractions (38). Interestingly, the recovery of Ca transients can be prevented by the CaMKII inhibitor KN-93 and it was proposed that CaMKII-dependent PLB phosphorylation may be responsible for the faster $[\mathrm{Ca}]_{\mathrm{i}} \mathrm{de}-$ cline and recovery of contractions that partially overcome the direct inhibitory effect of acidosis $(38,39)$. This is consistent with an inhibition of phosphatases during acidosis and increased PLB phosphorylation (40). We recently confirmed the importance of PLB phosphorylation by CaMKII in the absence of PKA phosphorylation in mouse myocytes (41). We showed also that PLB is required for the recovery of $\Delta[\mathrm{Ca}]_{\mathrm{i}}$ during acidosis using isolated myocytes from PLB knockout compared to wild-type mice. In addition, KN-93 inhibited CaMKII-dependent protein phosphorylation (e.g., PLB Thr17). Moreover, preliminary results from our laboratory have shown that acute overexpression of CaMKII in rabbit cardiac myocytes improves recovery during late acidosis leading to increased twitch shortening, $[\mathrm{Ca}]_{\mathrm{i}}$, as well as accelerated relaxation parameters (42).

\section{Arrhythmias}

Early afterdepolarizations (EAD) are thought to initiate long QT-arrhythmias. CaMKII was first thought to be involved in arrhythmias in a study by Anderson et al. (11). The authors found that KN-93 decreases the amount of EAD which they induced in isolated rabbit hearts and they showed that $\mathrm{I}_{\mathrm{Ca}}$ was the mechanism responsible. This was convincingly done by single cell studies showing that $\mathrm{I}_{\mathrm{Ca}}$ and RyR are needed to initiate EADs (13). In a follow-up study, the same group described increased arrhythmias in a mouse model of transgenic CaMKIV overexpression leading to hypertrophy (43).
Interestingly, in this mouse model increased CaMKII activity was found as a side effect. Electrophysiologically, this model is associated with increased AP duration, as also shown by specific overexpression of CaMKII (see below; Ref. 29) and QT prolongation leading to EAD. Arrhythmias could be increased by isoproterenol and decreased by $\mathrm{KN}-93$ and a specific inhibiting peptide for CaMKII.

Early reports show that, in addition to the Ca channels, also cardiac Na channels might be regulated by CaMKII possibly contributing to cardiac arrhythmias (44). Also, in human atrial myocytes, transient outward $\mathrm{K}$ current can be accelerated using KN-93 (45). In summary, the role of CaMKII in arrhythmias needs to be further investigated and the subcellular mechanisms need to be explored in detail.

\section{CaMKII and hypertrophy and heart failure}

In addition to the acute effects of CaMKII on ECC in the heart discussed in the previous sections, CaMKII is also involved in longer-term regulation of gene expression, termed excitation-transcription coupling, especially in hypertrophy and heart failure (2).

It has been known for several years that $\mathrm{Ca}$ can bind to $\mathrm{CaM}$ in different cell types which then translocate into the nucleus activating $\mathrm{CaMK}$, resulting in phosphorylation of the transcription factor cAMP response element binding protein (CREB) which promotes transcription of c-fos (3). CREB residue Ser-133 was the major site of phosphorylation by the CaMK in vitro and of phosphorylation after membrane depolarization in vivo (2). Interestingly, phosphorylation of Ser-142 seems to negatively regulate CREBdependent transcription. CaM might also be associated with the nuclear envelope. In the heart, overexpression of $\mathrm{CaM}$ in transgenic mice causes severe cardiac hypertrophy (46) 
and results in higher CaMKII phosphorylation (and activity), and the expression of the hypertrophic marker atrial natriuretic factor (Ref. 47). Interestingly, the CaM antagonist W-7 and the CaMKII antagonist KN-62 (48) could prevent hypertrophy in cultured myocytes, further implicating $\mathrm{Ca}-\mathrm{CaM}$ as a mediator of the hypertrophic response. Activated CaMKIV (and I) can also induce hypertrophic responses in cultured cardiomyocytes and transgenic mice (49). CaMKIV is expressed at very low levels in the heart $v s$ CaMKII, but like CaMKIII $\delta_{\mathrm{B}}$ it can enter the nucleus (1).

Recently, it was shown that CaMKII might also be involved in the transduction of hypertrophic stimuli through class II histone deacetylases (HDAC) which contain two CaMK phosphorylation sites and usually are bound to myocyte elongation factor 2 (MEF2), thereby inhibiting transcription (50). When HDAC are phosphorylated, dissociation from MEF2 and nuclear export of HDAC results in activation of MEF2 (51) which interacts with nuclear factor of activated $\mathrm{T}$ cells and GATA transcription factors leading to hypertrophic gene expression. Interestingly, HDAC translocation from the nucleus might be a very local process due to perinuclear $\mathrm{Ca}$ release (as a consequence of $\mathrm{IP}_{3}$ increase) independent of cytosolic $\mathrm{Ca}$ transients during ECC leading to local CaMKII activation, i.e., nuclear isoform $\mathrm{CaMKIII}_{\mathrm{B}}$ (52). In agreement with this are preliminary data showing $\mathrm{InsP}_{3}$ receptors concentrated in the nuclear envelope of cardiac myocytes which are associated with CaMKII $\delta$ (53).

New and exciting reports have also indicated that chronic $\beta$-adrenergic stimulation, in contrast to short-term stimulation which typically activates the cAMP/PKA pathway, activates CaMKII with subsequent stimulation of CaMKII-dependent pathways (54) and even apoptosis (55).

The first reports regarding human heart failure a few years ago described that CaMKII activity was increased almost 2-3-fold (2). It was suggested that this increase was compensatory because it correlated positively with the cardiac index and ejection fraction of patients with greatly impaired left ventricular function. In a different study, a 2fold increase in CaMKII $\delta$ was reported in failing human myocardium (2). Phosphatase activity is also enhanced in human heart failure (2), such that the net effect is unclear with respect to the phosphorylation state of individual target proteins. For example, while many PKA targets are relatively dephosphorylated in heart failure, the RyR may be hyperphosphorylated, a fact initially thought to be solely due to reduction of local phosphatase bound directly to the RyR (56).

Animal models of cardiac hypertrophy induced by transverse aortic constriction also showed an increase in CaMKII expression $(28,47)$ whereas CaMKI and IV expression levels were not changed (47). Ramirez et al. (57) reported that the specific nuclear isoform of CaMKII $\left(\delta_{\mathrm{B}}\right)$ caused transcriptional activation of atrial natriuretic factor gene expression (a hypertrophic signaling marker) in neonatal rat ventricular myocytes. Similarly, transgenic overexpression of $\mathrm{CaMKII}_{\mathrm{B}}$ in mice induces cardiac hypertrophy and modest ventricular dilation (58). A similar phenotype is induced by transgenic expression of CaMKIV (49), which also enters the nucleus. However, CaMKIV is expressed at very low levels in the heart $(2,4)$.

To investigate the effects of CaMKII $\delta$ on cellular Ca regulation we have overexpressed the cytoplasmic $\delta_{\mathrm{C}}$ isoform in mouse heart and reported in two accompanying papers the phenotype and cellular basis for hypertrophy and heart failure found in this model $(28,29)$. The transgenic line studied exhibited a 3 -fold increase in CaMKII $\delta$ activity, profound dilated hypertrophy and severe ventricular dysfunction (Figure 1). Since cytoplasmic CaMKII targets numerous $\mathrm{Ca}-$ handling proteins, we compared Ca regulation in cardiomyocytes isolated from 3- 
month-old transgenic CaMKII $\delta \mathrm{C}$ mice vs wild-type littermates. We found major alterations in intracellular $\mathrm{Ca}$ handling with marked reductions in twitch $\Delta[\mathrm{Ca}]_{\mathrm{i}}$ and $\mathrm{SR}$ $\mathrm{Ca}$ content (Figure 2). This was associated with decreased SERCA2 and PLB expression, but enhanced NCX function and expression, all typical features which are widely accepted for heart failure (37).

Interestingly, possible acute effects of
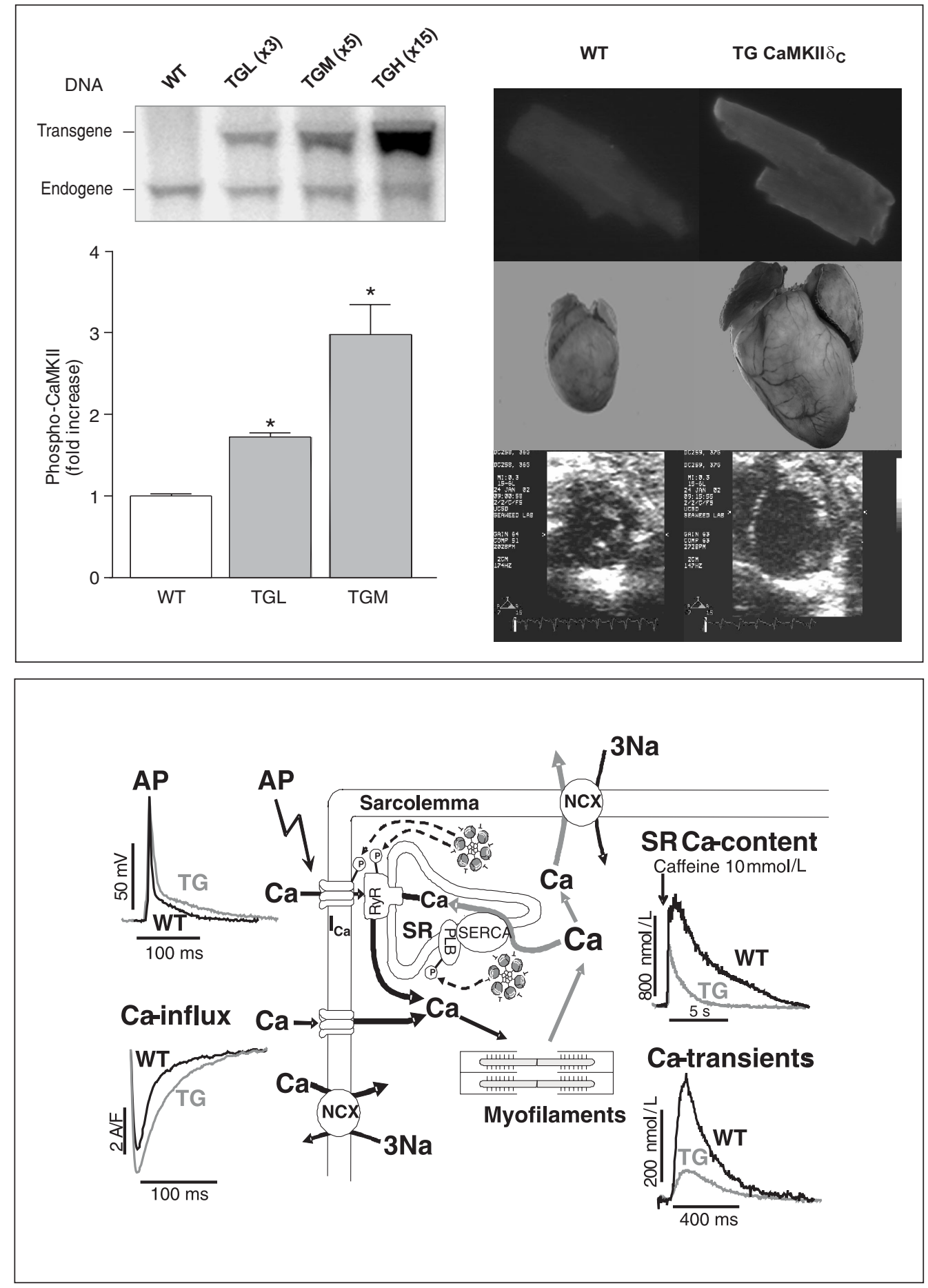

Figure 2. CaMKIII $\delta_{\mathrm{C}}$ overexpression and excitation-contraction coupling. CaMKII phosphorylates several Ca-handling proteins including phospholamban (PLB), SR Ca release channels (RyR), and L-type Ca channels responsible for $\mathrm{Ca}$ influx ( $\mathrm{I}_{\mathrm{Ca}}$ ). Action potential (AP) duration is prolonged, and $\mathrm{Ca}$ influx is increased. However, SR Ca content and $\mathrm{Ca}$ transients are greatly decreased leading to contractile dysfunction (adapted from Refs. 28 and 29). TG and $\mathrm{WT}=$ transgenic and wild-type mice, respectively; $\mathrm{NCX}=\mathrm{Na} /$ Ca-exchanger; SR = sarcoplasmic reticulum; SERCA $=$ SR CaATPase. 
CaMKII on RyR phosphorylation and function were also detected which do not fit the usual findings in heart failure. Thus, the frequency of $\mathrm{Ca}$ sparks (indicative of diastolic spontaneous SR Ca release events or

Figure 3. Elementary SR Ca release events (Ca sparks). Original confocal images showing significantly increased Ca spark frequency in CaMKIII $\delta_{C}$ transgenic (TG) mouse myocytes leading to a CaMKII-dependent diastolic SR Ca leak. This leak is significantly ( 4 times) higher in TG than in wild type (WT). In contrast, CaMKII inhibition using $\mathrm{KN}-93$ decreases $\mathrm{Ca}$ spark frequency back to control levels. An explanation for this increased $\mathrm{Ca}$ spark frequency most likely is the CaMKII-dependent hyperphosphorylation of SR Ca release channels (RyR; adapted from Refs. 28 and 29). $\mathrm{CaMKII}=\mathrm{Ca} /$ calmodulin-dependent protein kinase II; au $=$ arbitrary units. ${ }^{*} \mathrm{P}<0.05$ compared to WT.

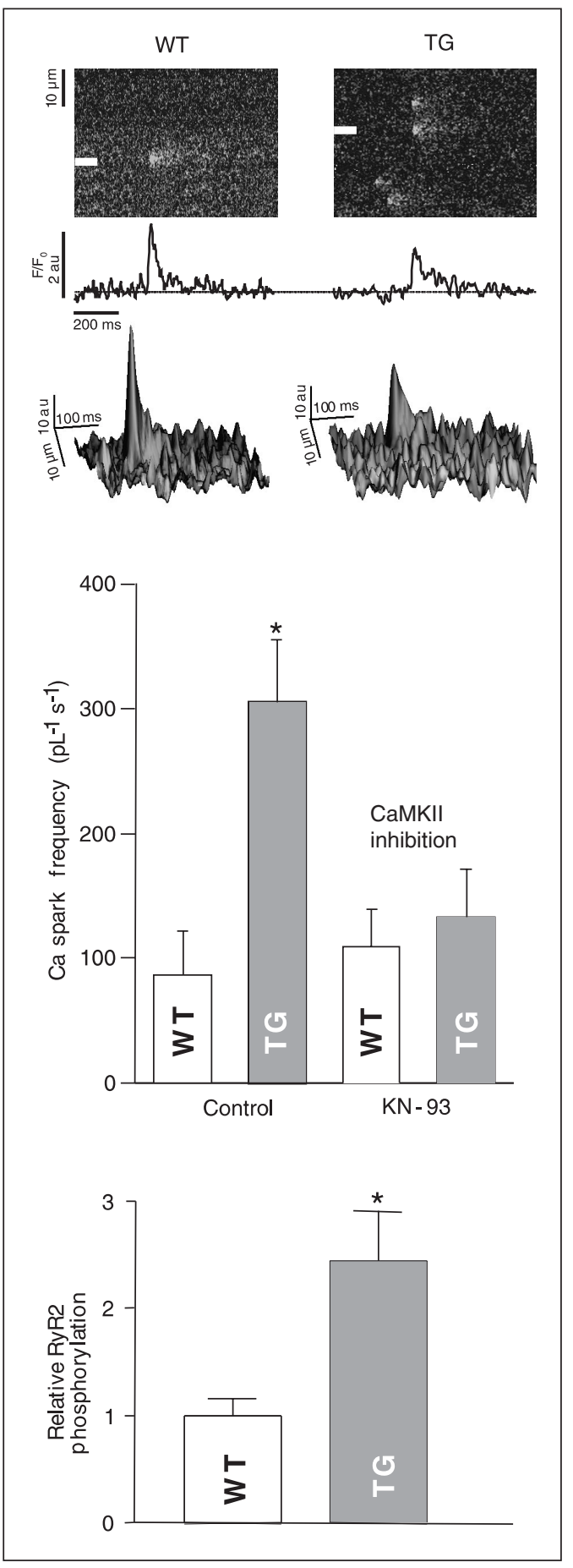

opening of RyR clusters), width, and duration were greatly enhanced, demonstrating increased diastolic SR Ca leak (Figure 3). Therefore, in addition to disturbed SR Ca uptake, increased $\mathrm{Ca}$ loss from the SR by means of a leaky RyR might be important in CaMKII-induced heart failure. Also, the increased Ca spark frequency was interesting since this is usually decreased when SR Ca load is reduced. We found that this most likely is due to increased RyR phosphorylation directly by CaMKII, resulting in increased and prolonged openings of the RyR. Most importantly, the increased $\mathrm{Ca}$ spark frequency could be reduced back to normal levels by blocking CaMKII with KN-93, and backphosphorylation indeed showed increased RyR phosphorylation in transgenic compared to wild-type mice. These results showing a novel mechanism of CaMKIIdependent SR Ca leak through hyperphosphorylated RyR in heart failure were recently confirmed by two independent groups. They showed that the peptide inhibitor AIP depresses Ca spark frequency in rabbit hearts due to decreased CaMKII binding to RyR (30) and that CaMKII-dependent RyR phosphorylation increases RyR open probability using single channel measurements in lipid bilayers (31). Reduced contractile function in this model of heart failure could be explained by a combination of higher SR $\mathrm{Ca}$ leak, weaker SR Ca pumping, and greater NCX function due to RyR hyperphosphorylation.

\section{Final considerations}

CaMKII has regained attention during the last few years. Its involvement in cardiac hypertrophy and heart failure is novel and interesting. New mechanisms were proposed and confirmed by independent groups. Exact hypertrophic signaling pathways still need to be investigated, but a couple of internationally well-recognized groups are on their way. Also, the new findings of CaMKII in 
apoptosis and arrhythmias open a new field of research. We are just starting to understand the subcellular mechanisms responsible for it. The next few years will bring more insight into these mechanisms and possibly therapeutic approaches on experimental levels might be initiated thereafter.

\section{Acknowledgments}

I would cordially like to thank FESBE for the invitation to this meeting and Stefan Wagner, Michael Kohlhaas, and Can-Martin Sag, University of Göttingen, Göttingen, Germany, for comments on the manuscript.

\section{References}

1. Braun AP \& Schulman $H$ (1995). The multifunctional calcium/calmodulin-dependent protein kinase: from form to function. Annual Review of Physiology, 57: 417-445.

2. Maier LS \& Bers DM (2002). Calcium, calmodulin, and calciumcalmodulin kinase II: heartbeat to heartbeat and beyond. Journal of Molecular and Cellular Cardiology, 34: 919-939.

3. Hook SS \& Means AR (2001). $\mathrm{Ca}^{2+} / \mathrm{CaM}$-dependent kinases: From activation to function. Annual Review of Pharmacology and Toxicology, 41: 471-505.

4. Zhang $\mathrm{T}$ \& Brown JH (2004). Role of $\mathrm{Ca}^{2+} /$ calmodulin-dependent protein kinase II in cardiac hypertrophy and heart failure. Cardiovascular Research, 63: 476-486.

5. Meyer T, Hanson PI, Stryer L et al. (1992). Calmodulin trapping by calcium-calmodulin-dependent protein kinase. Science, 256: 11991202.

6. Miller SB \& Kennedy MB (1986). Regulation of brain type II Ca/ calmodulin-dependent protein kinase by autophosphorylation: A Catrigger molecular switch. Cell, 44: 861-870.

7. Lai $Y$, Nairn AC \& Greengard $P$ (1986). Autophosphorylation reversibly regulates the $\mathrm{Ca} / \mathrm{calmodulin}$-dependent protein kinase II. Proceedings of the National Academy of Sciences, USA, 83: 42534257.

8. Lou LL, Lloyd SJ \& Schulman H (1986). Activation of the multifunctional $\mathrm{Ca} / \mathrm{calmodulin}$-dependent protein kinase by autophosphorylation: ATP modulates production of an autonomous enzyme. Proceedings of the National Academy of Sciences, USA, 83: 94979501.

9. Schworer CM, Colbran RJ \& Soderling TR (1986). Reversible generation of a Ca-independent form of $\mathrm{Ca}$ (calmodulin)-dependent protein kinase II by an autophosphorylation mechanism. Journal of Biological Chemistry, 261: 8581-8584.

10. Li L, Satoh H, Ginsburg KS et al. (1997). The effect of Ca-calmodulin-dependent protein kinase II on cardiac excitation-contraction coupling in ferret ventricular myocytes. Journal of Physiology, 501: 17-32.

11. Anderson ME, Braun AP, Wu Y et al. (1998). KN-93, an inhibitor of multifunctional $\mathrm{Ca}^{++} /$calmodulin-dependent protein kinase, decreases early afterdepolarizations in rabbit heart. Journal of Pharmacology and Experimental Therapeutics, 287: 996-1006.

12. Ishida A, Kameshita I, Okuno S et al. (1995). A novel specific and potent inhibitor of calmodulin-dependent protein kinase II. Biochemical and Biophysical Research Communications, 212: 806-812.

13. Wu Y, MacMillan LB, McNeill RB et al. (1999). CaM kinase augments cardiac L-type $\mathrm{Ca}^{2+}$ current: a cellular mechanism for long QT arrhythmias. American Journal of Physiology, 276: H2168-H2178.

14. Bers DM (2002). Cardiac excitation-contraction coupling. Nature, 415: 198-205.
15. Witcher DR, Kovacs RJ, Schulman $\mathrm{H}$ et al. (1991). Unique phosphorylation site on the cardiac ryanodine receptor regulates calcium channel activity. Journal of Biological Chemistry, 266: 11144-11152.

16. Hain J, Onoue H, Mayrleitner M et al. (1995). Phosphorylation modulates the function of the calcium release channel of sarcoplasmic reticulum from cardiac muscle. Journal of Biological Chemistry, 270: 2074-2081.

17. Davis BA, Schwartz A, Samaha FJ et al. (1983). Regulation of cardiac sarcoplasmic reticulum calcium transport by calcium-calmodulin-dependent phosphorylation. Journal of Biological Chemistry, 258: 13587-13591.

18. Simmerman HKB, Collins JH, Theibert JL et al. (1986). Sequence analysis of PLB: Identification of phosphorylation sites and two major structural domains. Journal of Biological Chemistry, 261: 13333-13341.

19. Lee KS (1987). Potentiation of the calcium-channel currents of internally perfused mammalian heart cells by repetitive depolarization. Proceedings of the National Academy of Sciences, USA, 84: 3941-3945.

20. Hryshko LV \& Bers DM (1990). Ca current facilitation during postrest recovery depends on Ca entry. American Journal of Physiology, 259: H951-H961.

21. Yuan W \& Bers DM (1994). Ca-dependent facilitation of cardiac Ca current is due to Ca-calmodulin dependent protein kinase. American Journal of Physiology, 267: H982-H993.

22. Anderson ME, Braun AP, Schulman $\mathrm{H}$ et al. (1994). Multifunctional $\mathrm{Ca} /$ calmodulin-dependent protein kinase mediates Ca-induced enhancement of the L-type $\mathrm{Ca}$ current in rabbit ventricular myocytes. Circulation Research, 75: 854-861.

23. Xiao RP, Cheng H, Lederer WJ et al. (1994). Dual regulation of $\mathrm{Ca} /$ calmodulin kinase II activity by membrane voltage and by calcium influx. Proceedings of the National Academy of Sciences, USA, 91: 9659-9663.

24. Dzhura I, Wu Y, Colbran RJ et al. (2000). Calmodulin kinase determines calcium-dependent facilitation of L-type calcium channels. Nature Cell Biology, 2: 173-177.

25. Lokuta AJ, Rogers TB, Lederer WJ et al. (1997). Modulation of cardiac ryanodine receptors of swine and rabbit by a phosphorylation-dephosphorylation mechanism. Journal of Physiology, 487: 609622.

26. duBell WH, Lederer WJ \& Rogers TB (1996). Dynamic modulation of excitation-contraction coupling by protein phosphatase in rat ventricular myocytes. Journal of Physiology, 493: 793-800.

27. Wu Y, Colbran RJ \& Anderson ME (2001). Calmodulin kinase is a molecular switch for cardiac excitation-contraction coupling. Proceedings of the National Academy of Sciences, USA, 98: 28772881. 
28. Zhang T, Maier LS, Dalton ND et al. (2003). The $\delta C$ isoform of CaMKII is activated in cardiac hypertrophy and induces dilated cardiomyopathy and heart failure. Circulation Research, 92: 912919.

29. Maier LS, Zhang T, Chen L et al. (2003). Transgenic CaMKII $\delta C$ overexpression uniquely alters cardiac myocyte $\mathrm{Ca}^{2+}$ handling: $\mathrm{Re}-$ duced $\mathrm{SR} \mathrm{Ca}^{2+}$ load and activated $\mathrm{SR} \mathrm{Ca}^{2+}$ release. Circulation Research, 92: 904-911.

30. Currie S, Loughrey CM, Craig MA et al. (2004). Calcium/calmodulindependent protein kinase II $\delta$ associates with the ryanodine receptor complex and regulates channel function in rabbit heart. Biochemical Journal, 377: 357-366.

31. Wehrens XH, Lehnart SE, Reiken SR et al. (2004). $\mathrm{Ca}^{2+} /$ calmodulin-dependent protein kinase II phosphorylation regulates the cardiac ryanodine receptor. Circulation Research, 94: e61-e70.

32. Brittsan AG \& Kranias EG (2000). Phospholamban and cardiac contractile function. Journal of Molecular and Cellular Cardiology, 32: 2131-2139.

33. Bassani RA, Mattiazzi A \& Bers DM (1995). CaMKII is responsible for activity-dependent acceleration of relaxation in rat ventricular myocytes. American Journal of Physiology, 268: H703-H712.

34. Hagemann D, Kuschel M, Kuramochi T et al. (2000). Frequencyencoding Thr17 phospholamban phosphorylation is independent of Ser16 phosphorylation in cardiac myocytes. Journal of Biological Chemistry, 275: 22532-22536.

35. Schouten VJ (1990). Interval dependence of force and twitch duration in rat heart explained by Ca pump inactivation in sarcoplasmic reticulum. Journal of Physiology, 431: 427-444.

36. DeSantiago J, Maier LS \& Bers DM (2002). Frequency-dependent acceleration of relaxation in the heart depends on CaMKII, but not phospholamban. Journal of Molecular and Cellular Cardiology, 34: 975-984.

37. Bers DM (2001). Excitation-Contraction Coupling and Cardiac Contractile Force. 2nd edn. Kluwer Academic Publishers, Dordrecht, The Netherlands.

38. Nomura N, Satoh H, Terada H et al. (2002). CaMKIl-dependent reactivation of $\mathrm{SR} \mathrm{Ca}^{2+}$ uptake and contractile recovery during intracellular acidosis. American Journal of Physiology, 283: H193H203.

39. Komukai K, Pascarel C \& Orchard CH (2001). Compensatory role of CaMKII on $\mathrm{I}_{\mathrm{Ca}}$ and $\mathrm{SR}$ function during acidosis in rat ventricular myocytes. Pflügers Archiv, 442: 353-361.

40. Vittone L, Mundiña-Weilenmann C, Said M et al. (1998). Mechanisms involved in the acidosis enhancement of the isoproterenolinduced phosphorylation of phospholamban in the intact heart. Journal of Biological Chemistry, 273: 9804-9811.

41. DeSantiago J, Maier LS \& Bers DM (2004). Phospholamban is required for CaMKII-dependent recovery of $\mathrm{Ca}$ transients and SR Ca reuptake during acidosis in cardiac myocytes. Journal of Molecular and Cellular Cardiology, 36: 67-74.

42. Sag CM, DeSantiago J, Bers DM et al. (2004). Recovery of Ca transients, SR Ca reuptake and shortening during acidosis requires the CaMKII/PLB signaling pathway but is impaired in heart failure. European Heart Journal, 25 (Suppl): 179 (Abstract).

43. Wu Y, Temple J, Zhang R et al. (2002). Calmodulin kinase II and arrhythmias in a mouse model of cardiac hypertrophy. Circulation, 106: $1288-1293$
44. Wagner S, Rasenack ECL, Jacobshagen C et al. (2004). Ca/calmodulin-dependent protein kinase II (CaMKII) reduces the availability of cardiac voltage-gated Na channels. Circulation, 110 (Suppl III): 61 (Abstract)

45. Tessier S, Karczewski P, Krause EG et al. (1999). Regulation of the transient outward $\mathrm{K}^{+}$current by $\mathrm{Ca}^{2+} /$ calmodulin-dependent protein kinases II in human atrial myocytes. Circulation Research, 85: 810819

46. Gruver CL, DeMayo F, Goldstein MA et al. (1993). Targeted developmental overexpression of calmodulin induced proliferative and hypertrophic growth of cardiomyocytes in transgenic mice. Endocrinology, 133: 376-388.

47. Colomer JM \& Means AR (2000). Chronic elevation of calmodulin in the ventricles of transgenic mice increases the autonomous activity of calmodulin-dependent protein kinase II, which regulates atrial natriuretic factor gene expression. Molecular Endocrinology, 14: 1125-1136.

48. Zhu W, Zou Y, Shiojima I et al. (2000). $\mathrm{Ca}^{2+} /$ calmodulin-dependent kinase II and calcineurin play critical roles in endothelin-1-induced cardiomyocyte hypertrophy. Journal of Biological Chemistry, 275: 15239-15245

49. Passier R, Zeng $\mathrm{H}$, Frey $\mathrm{N}$ et al. (2000). CaM kinase signaling induces cardiac hypertrophy and activates the MEF2 transcription factor in vivo. Journal of Clinical Investigation, 105: 1395-1406.

50. McKinsey TA, Zhang CL \& Olson EN (2002). MEF2: a calciumdependent regulator of cell division, differentiation and death. Trends in Biochemical Sciences, 27: 40-47.

51. McKinsey TA, Zhang CL, Lu J et al. (2000). Signal-dependent nuclear export of a histone deacetylase regulates muscle differentiation. Nature, 408: 106-111.

52. Wu X, Bossuyt J, Zhang T et al. (2004). Excitation-transcription coupling in adult myocytes: Local InsP3-dependent perinuclear signaling activates HDAC nuclear export. Circulation, 110: II-285 (Abstract).

53. Bare DJ, Bers DM \& Mignery GA (2004). InsP3 receptors in ventricular myocytes are primarily in the nuclear envelope, associate with CaMKII $\delta$, and are phosphorylation targets. Circulation, 110: II-159 (Abstract)

54. Wang W, Zhu W, Wang S et al. (2004). Sustained B1-adrenergic stimulation modulates cardiac contractility by $\mathrm{Ca}^{2+} /$ calmodulin $\mathrm{ki}-$ nase signaling pathway. Circulation Research, 95: 798-806.

55. Zhu WZ, Wang SQ, Chakir K et al. (2003). Linkage of beta1-adrenergic stimulation to apoptotic heart cell death through protein kinase A-independent activation of $\mathrm{Ca}^{2+} /$ calmodulin kinase II. Jounal of Clinical Investigation, 111: 617-625.

56. Marx SO, Reiken S, Hisamatsu Y et al. (2000). PKA phosphorylation dissociates FKBP12.6 from the calcium release channel (ryanodine receptor): defective regulation in failing hearts. Cell, 101: 365-376.

57. Ramirez MT, Zhao XL, Schulman $H$ et al. (1997). The nuclear $\delta_{B}$ isoform of $\mathrm{Ca}$ /calmodulin-dependent protein kinase II regulates atrial natriuretic factor gene expression in ventricular myocytes. Journal of Biological Chemistry, 272: 31203-31208.

58. Zhang T, Johnson EN, Gu Y et al. (2002). The cardiac-specific nuclear $\delta_{\mathrm{B}}$ isoform of $\mathrm{Ca}^{2+} /$ calmodulin-dependent protein kinase II induces hypertrophy and dilated cardiomyopathy associated with increased protein phosphatase 2A activity. Journal of Biological Chemistry, 277: 1261-1267. 